\title{
Imaging of Polyethylene Glycol Layers on Nanoparticles
}

\author{
Sarah R. Anderson ${ }^{1}$, Mackensie C. Smith ${ }^{2}$, Jeffrey D. Clogston ${ }^{2}$, Anil K. Patri ${ }^{2}$, Scott E. McNeil ${ }^{2}$, and \\ Ulrich Baxa $^{1}$
}

1. Electron Microscopy Laboratory, and 2. Nanotechnology Characterization Laboratory, Cancer Research Technology Program, Leidos Biomedical Research, Inc., Frederick National Laboratory for Cancer Research, Frederick, MD 21702

The encapsulation of nanoparticles by adding polyethylene glycol (PEG) to their surface (PEGylation) is an important method to protect them from the immune system and from uptake by the reticuloendothelial system [1]. The addition of PEG creates nanoparticles with so-called "stealth" behavior that have significantly increased circulation times, reduced aggregation behavior, and less interaction with non-targeted serum and tissue proteins [2]. The PEG coating also increases solubility of nanoparticles in serum and buffer due to the long hydrophilic ethylene glycol repeats. The length of the PEG chain can be chosen according to application and the ends are usually modified for nanoparticle attachment on one end and often have a methoxy group on the other end.

Characterizing the PEG coating on nanoparticles is an essential part of nanoparticle synthesis optimization and quality control. There are several bulk methods to check the presence of PEG on nanoparticles including UV-vis, DLS, and zeta potential to name a few. But imaging the PEG layer directly on a particle by particle basis in the electron microscope has been a challenge. The PEG layer is basically invisible under most conditions for drop-cast nanoparticles and for cryo-EM specimens. Here we show that negative stain does not enable visualization of PEG layers in spite of several claims in the literature. However, the commercial availability of PEG antibodies (e.g. from Life Diagnostics, Inc.) allows the application of traditional IEM methods for the routine visualization of PEG layers on nanoparticles.

Colloidal gold nanoparticles, citrate-stabilized, and grafted with $10 \mathrm{kDa}$ and $20 \mathrm{kDa}$ mPEG have been imaged by traditional negative staining methods using uranyl acetate, uranyl formate, and methylamine tungstate $(\mathrm{NanoW})$. Weak coronas have been observed around many of the particles including the nanoparticles without PEG. The appearance of the corona is strongly dependent on staining conditions and depth and usually appears dark (more stain around gold particle) but under some conditions appears bright (less stain around gold nanoparticle). However, we show the presence of such coronas in all samples including the gold nanoparticles without PEG (Figure 1). Such coronas have been used in the literature as evidence of the presence of PEG on nanoparticle surfaces. However, based on our experiments we conclude that negative staining is not a reliable method to verify presence and size of the PEG layer.

Traditional solid phase IEM methods have been used to visualize the PEG layer of gold nanoparticles and metal oxide nanoparticles. Particles were applied to carbon film and imaged after a common IEM protocol. The IEM reaction was highly specific and had very little background (Figure 2). Care must be taken to separate any free PEG from the nanoparticles before application to the surface. 
In summary, we find IEM to be an easy, reliable, and reproducible method to visualize PEG layers on nanoparticles. Negative staining on the other hand was shown to not visualize the PEG layer and is at best considered highly unreliable.

\section{References}

[1] Jokerst JV, Lobovkina T, Zare RN, and Gambhir SS, Nanomed 6 (2011), p. 715 - 728.

[2] van Vlerken LE, Vyas TK, and Amiji MM, Pharm. Res. 24 (2007), p. 1405 - 1414.

[3] This project has been funded with Federal funds from the Frederick National Laboratory for Cancer Research, National Institutes of Health, under contract HHSN261200800001E. The content of this publication does not necessarily reflect the views or policies of the Department of Health and Human Services, nor does mention of trade names, commercial products or organizations imply endorsement by the US Government.
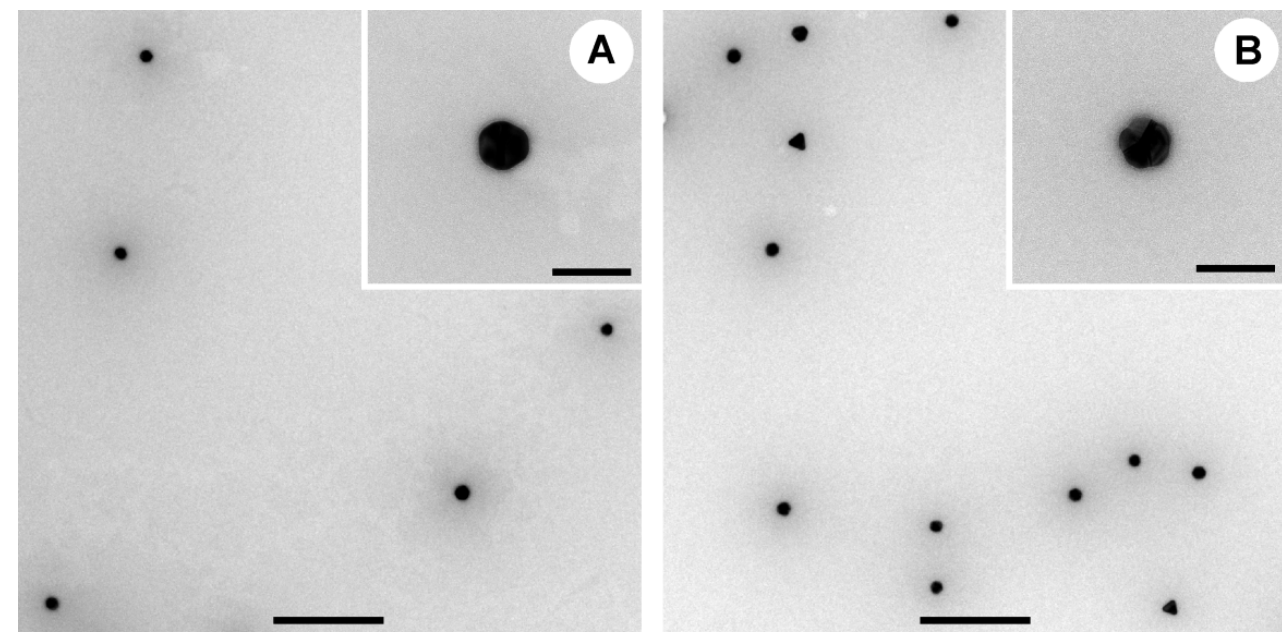

Figure 1. Negative staining with uranyl acetate of 60 nm gold nanoparticles: (A) without PEG. (B) coated with $10 \mathrm{kDa}$ PEG. Bar = $500 \mathrm{~nm}$ in main panel and bar $=100 \mathrm{~nm}$ in insert. The presence of PEG on these particles has been verified with DLS and Zeta potential measurements.
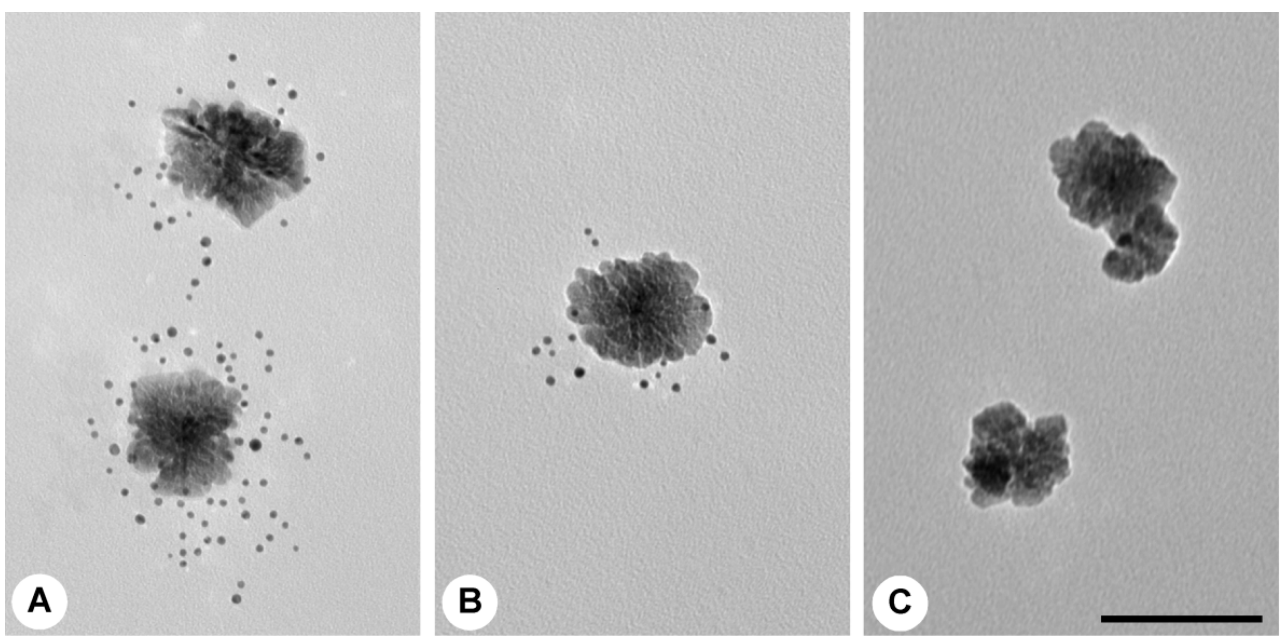

Figure 2. IEM of metal oxide particles using $10 \mathrm{~nm}$ gold labels: (A) antibody specific to PEG main chain (ethylene glycol moiety). (B) antibody specific to the methoxy end of PEG chain. (C) control = no primary antibody. Bar $=100 \mathrm{~nm}$. 